\title{
PRECISION TESTS OF QCD AT HERA*
}

\author{
NATAŠA RAIČEVIĆ \\ for the $\mathrm{H} 1$ and ZEUS collaborations \\ Faculty of Science, University of Montenegro \\ Džordža Vašingtona bb, 81000 Podgorica, Montenegro
}

(Received July 11, 2013)

\begin{abstract}
A brief overview is given on most recent results obtained by the HERA experiments, $\mathrm{H} 1$ and ZEUS, on the measurements providing information on parton density functions (PDFs) in the proton and on the strong coupling constant $\alpha_{\mathrm{s}}\left(M_{Z}\right)$. These measurements have very high experimental accuracy and can provide means for high precision tests of QCD.
\end{abstract}

DOI:10.5506/APhysPolBSupp.6.985

PACS numbers: 25.30.Dh, 12.15.Mm, 13.87.-a

\section{Introduction}

At the HERA collider, a center-of-mass energy of $\sqrt{s}=319 \mathrm{GeV}$ was achieved by colliding electrons or positrons with protons. The large energy allowed a value of $Q^{2}$, the exchanged boson virtuality, to reach almost $10^{5} \mathrm{GeV}^{2}$. A value of the Bjorken scaling variable $x$ which represents the momentum fraction carried by the struck quark in the proton's infinite momentum frame, reaches down to almost $10^{-5}$.

Until the year 2000 (HERA-I period), the experiments H1 and ZEUS at HERA collected an integrated luminosity of about $120 \mathrm{pb}^{-1}$ each. Afterwords, HERA underwent a major upgrade aiming for higher luminosity and until 2007 (HERA-II period), HERA provided in total about $500 \mathrm{pb}^{-1}$ of $e^{ \pm} p$ collisions to each of the experiments.

The study of $e^{ \pm} p$ deep inelastic scattering (DIS) from HERA has contributed significantly to tests of the Standard Model (SM) of electroweak and strong interactions.

* Presented at the Workshop "Excited QCD 2013", Bjelašnica Mountain, Sarajevo, Bosnia-Herzegovina, February 3-9, 2013. 


\section{Inclusive DIS cross section measurements}

Inclusive $e p$ DIS neutral current (NC), $e^{ \pm}+p \rightarrow e^{ \pm^{\prime}}+X$, and charged current $(\mathrm{CC}), e^{ \pm}+p \rightarrow \nu_{e}\left(\bar{\nu}_{e}\right)+X$, cross section measurements may be used to determine the combined sea quark distribution functions and the valence quark distributions in the proton. A QCD analysis in the DGLAP formalism also allows the gluon momentum distribution in the proton to be determined from scaling violations.

Recently, both collaborations finalised the inclusive cross section measurements at high $Q^{2}$ with HERA-II data. Inclusive $e^{ \pm} p$ cross sections for $\mathrm{NC}$ and CC DIS scattering processes measured with the $\mathrm{H} 1$ detector are in the range of $Q^{2}$ between 60 and $50000 \mathrm{GeV}^{2}$, and Bjorken $x$ between 0.0008 and 0.65 [1]. The measurements are combined with earlier published H1 data to improve statistical precision. New measurements of NC cross section in $e^{+} p$ collisions from HERA-II period obtained by the ZEUS Collaboration cover the kinematic region $Q^{2} \geq 185 \mathrm{GeV}^{2}$ [2]. The $Q^{2}$ dependence of the new and recently published $\mathrm{NC}$ and $\mathrm{CC}$ cross section measurements from the two experiments is shown in the left plot of Fig. 1 [1-5]. These measurements illustrate the unified behaviour of the electromagnetic and the weak interactions at high $Q^{2}$. The cross sections are well described by the SM expectations.
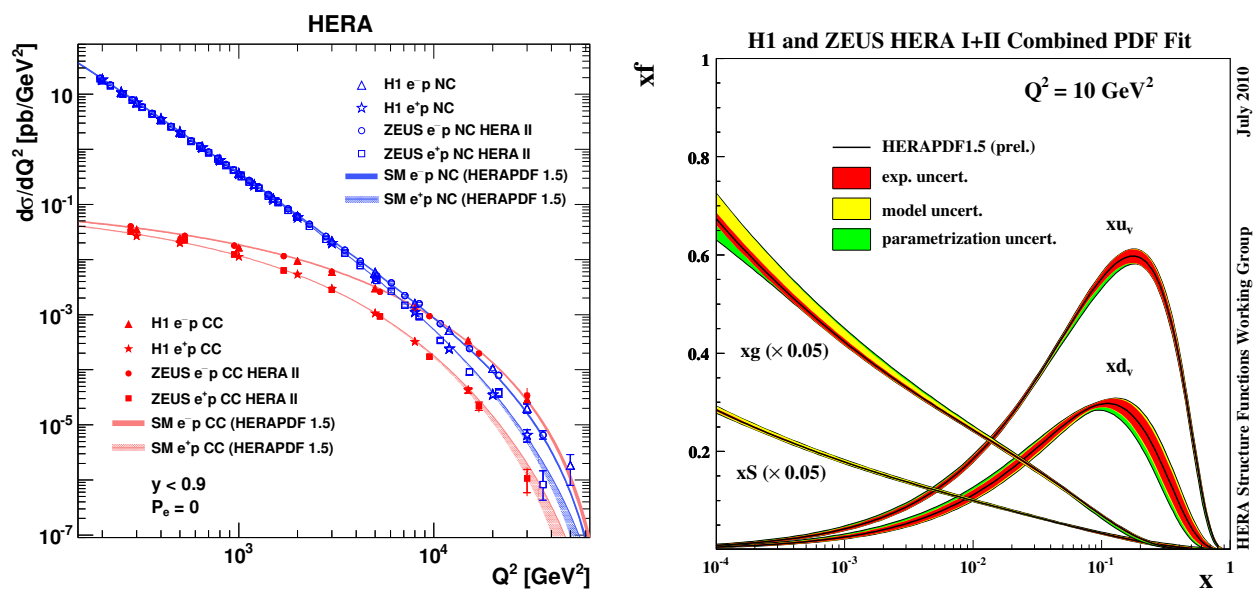

Fig. 1. $Q^{2}$ dependence of the NC and CC cross section measurements from $e^{-} p$ and $e^{+} p$ data at high $Q^{2}$ at HERA (left). The SM expectations are also shown as shaded bands. The inner and outer error bars represent the statistical and total errors, respectively. PDFs from HERAPDF1.5 at the scale of $10 \mathrm{GeV}^{2}$ (right). The gluon and sea distributions are scaled by a factor 0.05 . The experimental, model and parametrisation uncertainties are shown separately. 
The H1 and ZEUS collaborations have performed QCD fits to extract PDFs using HERA data alone. The fits are based on the evolution of the PDFs with $Q^{2}$ using DGLAP equations in Next-to-Leading Order (NLO). To achieve high precision measurements, data from both experiments have been combined leading to significantly reduced experimental uncertainties. The cross section measurements from NC and CC interactions from the HERA-I running period were combined and this combination was used for the extraction of the HERAPDF1.0 NLO parton density functions [6].

The effect of including HERA-II high $Q^{2}$ data for the determination of PDFs is analysed combining HERA-I and preliminary high $Q^{2}$ HERA-II data sets used as input to a PDF fit termed HERAPDF1.5 [7]. Inclusion of these data shows considerable improvement in the precision of the PDFs at high $x$, in the valence quark sector. Right plot of Fig. 1 is a summary plot for the valence distributions for up and down quarks as well as the gluon and sea quark distributions from HERAPDF1.5 at $Q^{2}=10 \mathrm{GeV}^{2}$.

\section{Charm production cross section measurement}

Charm quarks are predominantly produced by the boson-gluon fusion process, $\gamma g \rightarrow c \bar{c}$, which is sensitive to the gluon distribution in the proton. Measurements of open charm production via NC $e^{ \pm} p$ DIS scattering by the $\mathrm{H} 1$ and ZEUS experiments using different charm tagging methods were combined in the kinematic range of $2.5 \leq Q^{2} \leq 2000 \mathrm{GeV}^{2}$ and $3 \times 10^{-5} \leq$ $x \leq 5 \times 10^{-2}[8]$. The combined data are significantly more precise than any of the individual input data sets. This is an improvement of about a factor of 2 with respect to each of the most precise data sets in the combination.

The combined charm data together with the combined inclusive DIS cross sections from HERA are used as input for a detailed NLO QCD analysis based on different implementations of the variable flavour number scheme for the charm quark treatment. Inclusion of the new charm data allows for each scheme an optimal value of the charm mass parameter $\left(M_{c}^{\text {opt }}\right)$ to be determined as illustrated in the left plot of Fig. 2. The use of such parameter in the QCD analysis significantly reduces the uncertainties of PDFs, particularly for the sea quark contributions. In the right plot of Fig. 2, the sea anti-quarks $(x \bar{u}, x \bar{d}, x \bar{s}$ and $x \bar{c})$ PDFs from a fit using the inclusive HERA-I data only are compared with the corresponding PDFs when including the combined charm data in the fit. For both of these fits, the charm quark is treated according to the RT optimised scheme [9]. The uncertainty on the $x \bar{c}$ distribution function is considerably reduced and, consequently, the uncertainty on the $x \bar{u}$ distribution function is reduced because the inclusive data constrains the $u$-type anti-quark distribution $x \bar{U}=x \bar{u}+x \bar{c}$. Since the $x \bar{d}$ is constrained to be equal to $x \bar{u}$ at low $x$, the uncertainty on the $x \bar{d}$ distribution function is also reduced. 

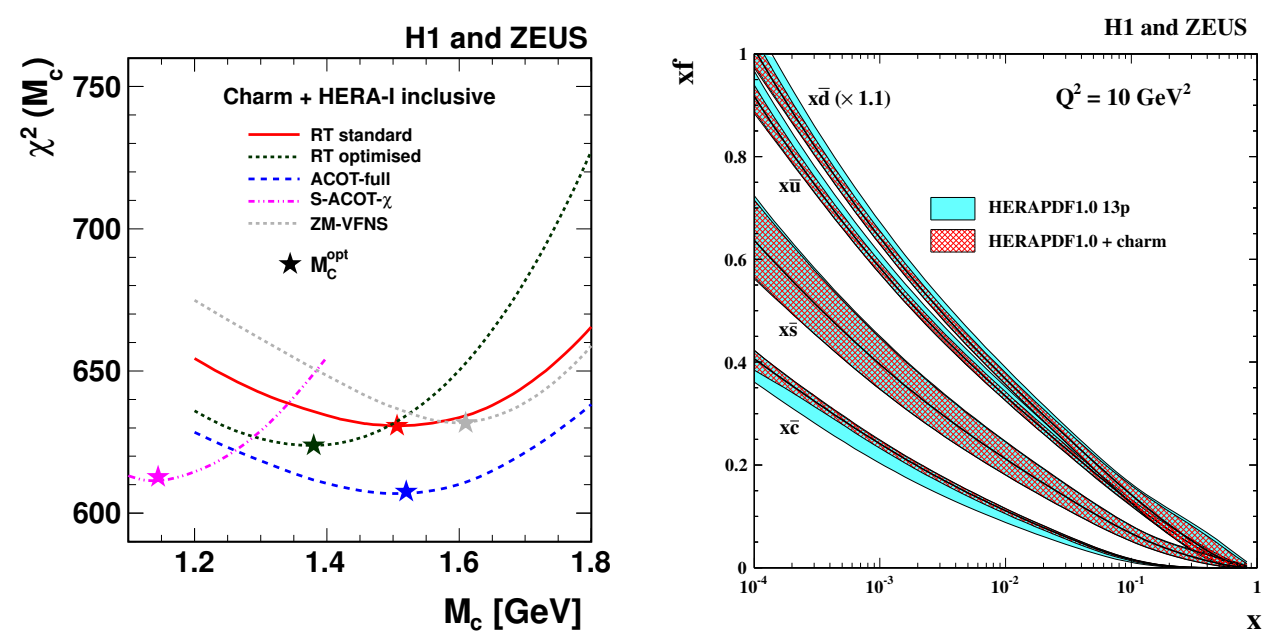

Fig. 2. The values of $\chi^{2}\left(M_{c}\right)$ for the QCD fit to the combined HERA inclusive DIS and charm measurements (left). Different heavy flavour schemes used in the fit are presented by lines with different styles. The values of $M_{c}^{\text {opt }}$ for each scheme are indicated by the stars. Sea anti-quarks obtained from the combined QCD analysis of the inclusive DIS data and charm data (dark shaded bands) in the RT optimised scheme [9] as a function of $x$ at $Q^{2}=10 \mathrm{GeV}^{2}$ (right). For comparison, the results of the QCD analysis of the inclusive DIS data only are also shown (light shaded bands). The $x \bar{d}$ distribution function is scaled by a factor 1.1 .

\section{Jet production}

The strong correlation between the gluon density function and strong coupling constant in the QCD analysis can be significantly reduced by the inclusion of jet data in the fit. The cross sections of jet production previously published by the H1 and ZEUS collaborations have been input to the HERAPDF1.6 QCD fit [10] in order to exploit their ability to constrain the gluon PDF and to make a determination of the value of the strong coupling constant simultaneously with the PDF determination. HERAPDF1.6 uses the same combined $\mathrm{H} 1$ and ZEUS inclusive DIS measurements as the HERAPDF1.5 fit. The value of $\alpha_{\mathrm{s}}$ with experimental, model, parametrisation, hadronisation and theory uncertainties extracted from the HERAPDF1.6 is

$$
\begin{aligned}
\alpha_{\mathrm{S}}\left(M_{Z}\right)=0.1202 & \pm 0.0013(\text { exp. }) \pm 0.0007(\bmod . / \text { par. }) \\
& \pm 0.0012(\text { had. })_{-0.0036}^{+0.0045}(\text { th. }) .
\end{aligned}
$$

New inclusive jet cross sections have been measured for photon virtuality $Q^{2}<1 \mathrm{GeV}^{2}$ and photon-proton centre-of-mass energies in the region $142<$ $W_{\gamma p}<293 \mathrm{GeV}$ with the ZEUS detector [11]. NLO QCD calculations 
give a good description of the measurements for the jet transverse energy $E_{\mathrm{T}}^{\mathrm{jet}}>21 \mathrm{GeV}$ as can be seen from the left plot of Fig. 3, in which the measured differential cross section $d \sigma / d \eta^{\text {jet }}$ is presented and compared to the NLO QCD calculation. The relative difference between the measured cross sections and the NLO QCD calculation is also shown. The shaded band displays the uncertainty due to the absolute energy scale of the jets and the hatched band displays the total theoretical uncertainty.
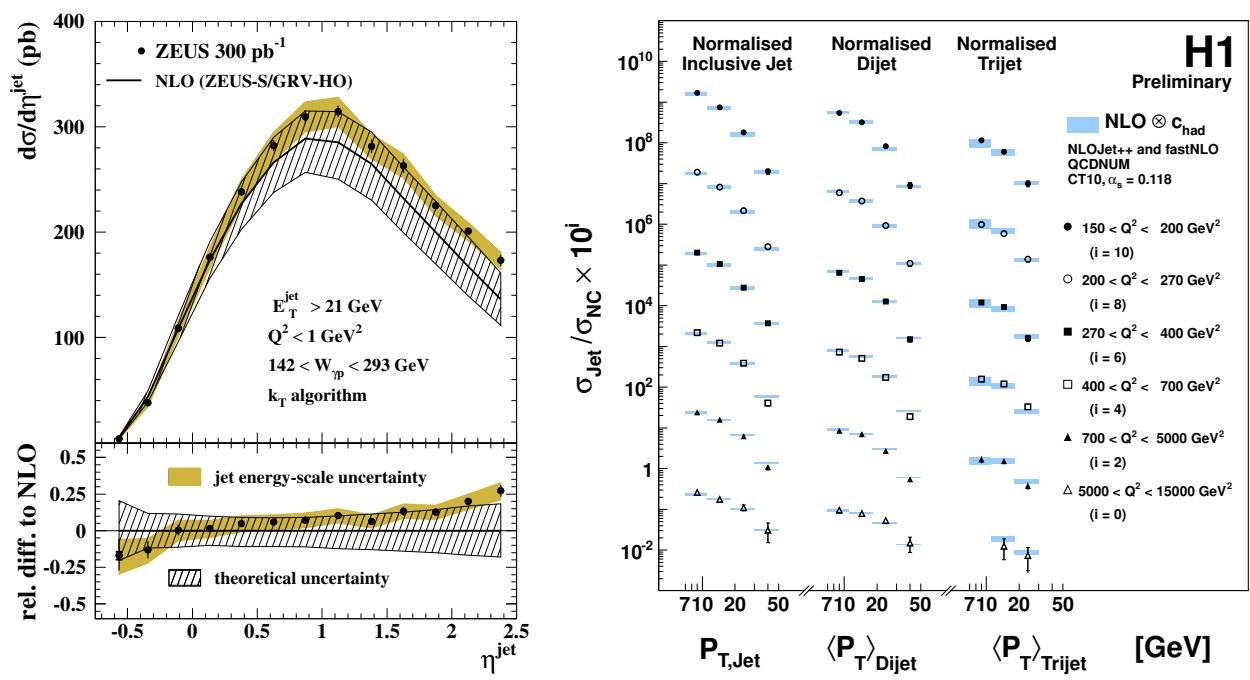

Fig. 3. The measured differential cross section $d \sigma / d \eta^{\text {jet }}$ for inclusive jet photoproduction compared to the NLO QCD calculation (left). Normalised inclusive jet cross sections as a functions of $Q^{2}$ and $P_{\mathrm{T}}$ and normalised dijet and trijet cross section as a function of $Q^{2}$ and $<P_{\mathrm{T}}>$ with comparison to NLO QCD predictions which are shown with the symmetrised theory uncertainties (right).

The measured cross sections were used to determine values of $\alpha_{\mathrm{S}}\left(M_{Z}\right)$. QCD fits to the cross sections for $21<E_{\mathrm{T}}^{\mathrm{jet}}<71 \mathrm{GeV}$ gave

$$
\alpha_{\mathrm{s}}\left(M_{Z}\right)=0.1206_{-0.0022}^{+0.0023}(\text { exp. })_{-0.0035}^{+0.0042} \text { (th.). }
$$

The H1 Collaboration made preliminary new results on normalised inclusive jet, dijet and trijet differential cross sections in neutral current DIS $e^{ \pm} p$ scattering based on a regularised unfolding procedure [12]. The inclusive jet (dijet, trijet) cross sections are measured in bins of $Q^{2}$ and the transverse momentum of a jet $P_{\mathrm{T}}\left(Q^{2}\right.$ and the average transverse momentum $\left.\left\langle P_{\mathrm{T}}\right\rangle\right)$ in the kinematic range of $150<Q^{2}<15000 \mathrm{GeV}^{2}$ and $-1.0<\eta^{\text {lab }}<2.5$. The cross sections are normalised to the inclusive DIS measurement in the corresponding bins of $Q^{2}$. The results are compared to NLO QCD predictions as shown in the right plot of Fig. 3. The NLO QCD calculations 
provide a good description of the measurements. Compared to a previously published result on normalised multijet cross sections, the new features are an extended range in jet pseudorapidity, an improved hadronic energy scale uncertainty of $1 \%$ and the adoption of a regularised unfolding procedure. The advantages of these normalised jet cross sections compared to absolute jet cross sections are reduced systematic experimental uncertainties as well as the PDF uncertainty.

The value of $\alpha_{\mathrm{s}}$ extracted from the simultaneous fit to the three normalised jet cross sections, with experimental, pdf, hadronisation and theory uncertainties is

$$
\left.\alpha_{\mathrm{S}}\left(M_{Z}\right)=0.1163 \pm 0.0008 \text { (exp.) } \pm 0.0014 \text { (pdf) } \pm 0.0011 \text { (had. }\right)_{-0.0035}^{+0.0044} \text { (th.) }
$$

The new results of $\alpha_{\mathrm{S}}\left(M_{Z}\right)$ agree well with the old measurements. The theoretical uncertainties are significantly larger than the experimental ones and are dominated by the uncertainty coming from higher orders not included in the calculations, determined conventionally by varying the renormalisation and factorisation scales by a factor of 2 up and down.

\section{REFERENCES}

[1] F.D. Aaron et al. [H1 Collaboration], J. High Energy Phys. 1209, 061 (2012).

[2] H. Abramowicz et al. [ZEUS Collaboration], arXiv:1208.6138 [hep-ex].

[3] H. Abramowicz et al. [ZEUS Collaboration], Eur. Phys. J. C70, 945 (2010).

[4] H. Abramowicz et al. [ZEUS Collaboration], Eur. Phys. J. C62, 625 (2009).

[5] H. Abramowicz et al. [ZEUS Collaboration], Eur. Phys. J. C61, 223 (2009).

[6] F.D. Aaron et al. [H1 and ZEUS collaborations], J. High Energy Phys. 1001, 109 (2010).

[7] H1 and ZEUS collaborations, H1prelim-10-142, ZEUS-prel-10-018 (2010).

[8] H. Abramowicz et al. [H1 and ZEUS collaborations], arXiv:1211.1182 [hep-ex].

[9] R.S. Thorne, Phys. Rev. D86, 074017 (2012) [arXiv:1201.6180 [hep-ph]].

[10] H1 and ZEUS collaborations, H1prelim-11-034, ZEUS-prel-11-001 (2011).

[11] H. Abramowicz et al. [ZEUS Collaboration], Nucl. Phys. B864, 1 (2012).

[12] H1 Collaboration, H1prelim-12-031 (2012). 